Document downloaded from:

http://hdl.handle.net/10251/161059

This paper must be cited as:

Singh, S.; Martínez Molada, E.; Maroju, P.; Behl, R. (2020). A study of the local convergence of a fifth order iterative method. Indian Journal of Pure and Applied Mathematics. 51(2):439455. https://doi.org/10.1007/s13226-020-0409-5

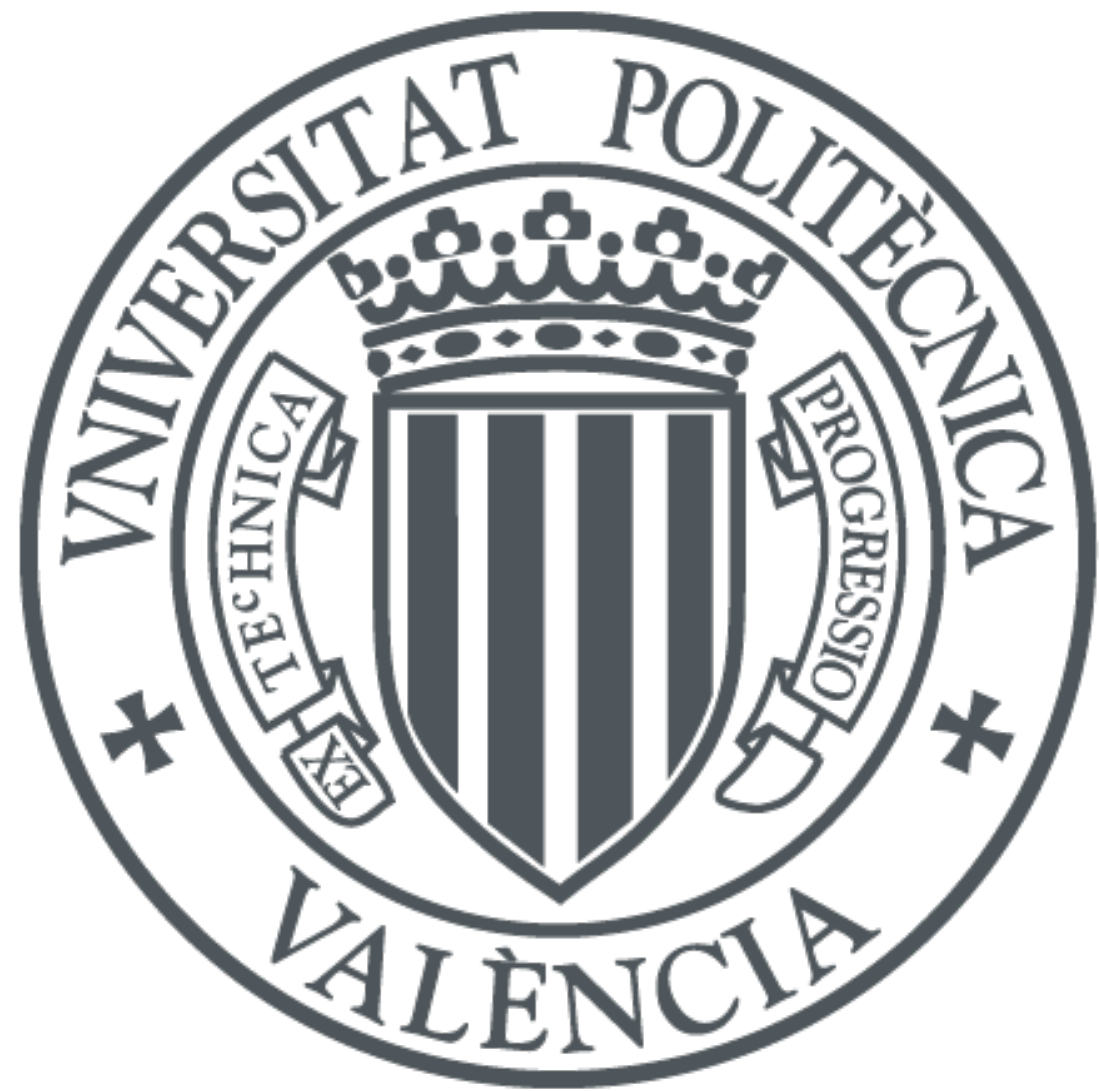

The final publication is available at

https://doi.org/10.1007/s13226-020-0409-5

Copyright Springer-Verlag

Additional Information 


\title{
A study of the local convergence of a fifth order iterative method
}

\author{
Sukjith Singh $^{\mathrm{a}}$, Eulalia Martínez ${ }^{\mathrm{b}}$, P. Maroju ${ }^{\mathrm{c}}$, R.Behl ${ }^{\mathrm{d}}$ \\ ${ }^{a}$ Dr. B.R. Ambedkar Nationl Instutute of Technology Jalandhar \\ ${ }^{b}$ Instituto Universitario de Matemática Multidisciplinar, Universitat Politècnica de València, Spain \\ ${ }^{c}$ Amrita School of Engineering, Department of Mathematics, Amrita Vishwa Vidhyapeetham, Coimbatore, India \\ ${ }^{d}$ Department of Mathematics, King Abdulaziz University, P.O. Box 80203, Jeddah 21589, Saudi Arabia
}

\begin{abstract}
We present a local convergence study of a fifth order iterative method to approximate a locally unique root of nonlinear systems. The analysis is discussed under the assumption that first order Fréchet derivative satisfies the Lipschitz continuity condition. Moreover, we consider the derivative free method obtained by approximating the derivative with divided difference along with the local convergence study. Finally, we provide computable radii and error bounds based on the Lipschitz constant for both cases analyzed in the theoretical results. Some of numerical examples are worked out and compared these results with existing methods results.
\end{abstract}

Keywords: Nonlinear equations, iterative methods, local convergence, divided differences. AMS Subject Classification: 65H05, 65H10.

\section{Introduction}

In this paper, we concerned with the problem of approximating a locally unique solution $\alpha$ of the nonlinear system

$$
F(x)=0
$$

Solving nonlinear system of equations play an important role in many branches of nonlinear Functional analysis, Numerical Analysis, Chemical engineering, Kinetic theory of gases [1, 2, 3, 4, 5, 6], etc. Many nonlinear problems arise from discretization of nonlinear integral equations and nonlinear differential equations by method of finite difference. In the literature, we can find several real world problems described by nonlinear models which can be transformed into system of nonlinear equations. Such nonlinear model are like variational inequalities, Bratu's problem, a shallow arch, etc. find in the paper [7]. However, most of the equations are phrased in terms of system of nonlinear equations of form (1).

The applicability of the nonlinear system on the problem of investigating coarse-grained dynamical properties of neuronal networks in kinetic theory discussed in [8]. In addition, Nejat and Ollivier [9] presented the problem to study the effect of discretization order on preconditioning and convergence of high-order Newton-Krylov unstructured flow solver in computational fluid dynamics. In [10], Grosan and Abraham showed the applicability of the system of nonlinear equations in neurophysiology, kinematics syntheses problem, chemical equilibrium problem, combustion problem and economics modeling

Email addresses: sukhjitmath@gmail.com (Sukjith Singh), eumarti@mat.up.es ( Eulalia Martínez), maroju.prashanth@gmail.com (P. Maroju), ramanbehl87@yahoo.in (R.Behl) 
problem. Very recently, Awawdeh and Tsoulos et al. [11, 12] solved the reactor and steering problems by phrasing them like systems of nonlinear equations.

There are many standard methods that can be used to approximate the solution of this kind of nonlinear equations. Main reason to develop iterative methods is that analytical solution often is not available for most type of nonlinear equations. These iterative methods divided into one point and multipoint methods, with memory and without memory methods are being studied nowadays. In fact, many higher order multipoint iterative methods for solving nonlinear equations are developed and published in various journals of applied and computer mathematics. We have well known quadratically convergent Newton's method for solving nonlinear equation (1), most of new methods use this one for first step to increase the order of convergence. Many authors have developed robust and efficient iterative methods with higher convergence order but it is very important to discuss the local and semilocal convergence analysis for them.

The study about local convergence of higher order iterative methods can be analyzed under different continuity conditions in Banach spaces (see, [13, 14]). Argyros and George [15] developed the local convergence analysis of third order Halley-like methods under Lipchitz continuity conditions and it is given for $k=0,1,2, \ldots$ by

$$
\begin{aligned}
y_{k} & =x_{k}-F^{\prime}\left(x_{k}\right)^{-1} F\left(x_{k}\right) \\
u_{k} & =y_{k}+(1-a) F^{\prime}\left(x_{k}\right)^{-1} F\left(x_{k}\right) \\
z_{k} & =y_{k}-\gamma A_{a, k} F^{\prime}\left(x_{k}\right)^{-1} F\left(x_{k}\right) \\
x_{k+1} & =z_{k}-\eta B_{a, k} F^{\prime}\left(x_{k}\right)^{-1} F\left(z_{k}\right),
\end{aligned}
$$

where, $\eta, \gamma, a \in(-\infty, \infty)-\{0\}, H_{a, k}=\frac{1}{a} F^{\prime}\left(x_{k}\right)^{-1}\left(F^{\prime}\left(u_{k}\right)-F^{\prime}\left(x_{k}\right)\right), A_{a, k}=I-\frac{1}{2} H_{a, k}\left(I-\frac{1}{2} H_{a, k}\right)$, $B_{a, k}=I-H_{1, k}+H_{a, k}^{2}$. The local convergence of Chebyshev-Halley-type method discussed in [17] and it is given for $k=0,1,2 \ldots$ by

$$
\begin{aligned}
y_{k} & =x_{k}-F^{\prime}\left(x_{k}\right)^{-1} F\left(x_{k}\right) \\
z_{k} & =x_{k}-\left(1+\left(F\left(x_{k}\right)-2 \eta F\left(y_{k}\right)\right)^{-1} F\left(y_{k}\right)\right) F^{\prime}\left(x_{k}\right)^{-1} F\left(x_{k}\right) \\
x_{k+1} & =z_{k}-\left(F^{\prime}\left(x_{k}\right)+\bar{F}^{\prime \prime}\left(x_{k}\right)\left(z_{k}-x_{k}\right)\right)^{-1} F\left(z_{k}\right), \quad k \geq 0,
\end{aligned}
$$

where, $\bar{F}^{\prime \prime}\left(x_{k}\right)=2 F\left(y_{k}\right) F^{\prime}\left(x_{k}\right)^{2} F\left(x_{k}\right)^{-2}$ and $\eta$ is a parameter. The order of this family is at least five for any value of $\eta$ and for $\eta=1$, it is six.

In this paper, we analyze the local convergence of fifth order iterative method which is proposed in [16] under the condition that first order Fréchet derivative satisfying the Lipschitz continuity condition. The existence and uniqueness region of the solution is established. Numerical examples worked out and convergence balls for each of them are obtained. We compare these results with the convergence balls of existing methods (2) and (3). Also, we discuss the local convergence of derivative free iterative method obtained by approximating the derivative by divided differences. Some numerical examples worked out and the convergence regions computed.

This paper is divided into four sections and organized as follows. In Section 1, we form the introduction. The local convergence study is performed in Section 2. The existence and uniqueness region of the solution is derived along with some numerical examples. In Section 3, the local convergence of the derivative free iterative method is discussed and also the computation of existence and uniqueness region of solution with numerical examples. Finally, the conclusion forms Section 4. 


\section{Local convergence analysis}

In this section, we consider a fifth order iterative method proposed in [16] and its local convergence analysis under Lipschitz conditions on $F^{\prime}$. It is given for $k=0,1,2, \ldots$ by

$$
\begin{aligned}
y_{k} & =x_{k}-F^{\prime}\left(x_{k}\right)^{-1} F\left(x_{k}\right) \\
z_{k} & =x_{k}-2\left(F^{\prime}\left(x_{k}\right)+F^{\prime}\left(y_{k}\right)\right)^{-1} F\left(x_{k}\right) \\
x_{k+1} & =z_{k}-F^{\prime}\left(y_{k}\right)^{-1} F\left(z_{k}\right),
\end{aligned}
$$

where $x_{0}$ is the starting point. In [16], Cordero et al. presented the fifth order of convergence using Taylor series on higher order Fréchet derivative without obtaining the convergence balls. They also assumed that the starting point $x_{0}$ is sufficiently close to the solution without estimating this closeness. Now, we have addressed these problems using only first order Fréchet derivative.

Suppose that $B(v, \rho)$ and $\bar{B}(v, \rho)$ denote the open and closed balls, respectively with center $v$ and radius $\rho$. Let $F: D \subseteq X \rightarrow Y$ be a Fréchet differentiable operator defined on open domain $D$ such that for $\alpha \in D, L_{0}>0, L>0$ and for all $x, y \in D$, we have

$$
\begin{aligned}
& F(\alpha)=0, \quad F^{\prime}(\alpha)^{-1} \in L(Y, X), F^{\prime}(\alpha)^{-1} \neq 0 \\
& \left\|F^{\prime}(\alpha)^{-1}\left(F^{\prime}(x)-F^{\prime}(\alpha)\right)\right\| \leq L_{0}\|x-\alpha\|, \quad \forall x \in D \\
& \left\|F^{\prime}(\alpha)^{-1}\left(F^{\prime}(x)-F^{\prime}(y)\right)\right\| \leq L\|x-y\|, \quad \forall x, y \in B\left(\alpha, \frac{1}{L_{0}}\right) \subseteq D .
\end{aligned}
$$

Lemma 1. If the nonlinear operator $F$ satisfies the above assumptions, then for all $x \in B\left(\alpha, \frac{1}{L_{0}}\right)$, we have

$$
\begin{aligned}
\left\|F^{\prime}(\alpha)^{-1} F^{\prime}(x)\right\| & \leq 1+L_{0}\|x-\alpha\|, \\
\left\|F^{\prime}(\alpha)^{-1} F^{\prime}(\alpha+t(x-\alpha))\right\| & \left.\leq 1+L_{0}\|x-\alpha\| \quad \forall t \in\right] 0,1[, \\
\left\|F^{\prime}(\alpha)^{-1} F(x)\right\| & \leq\left(1+L_{0}\|x-\alpha\|\right)\|x-\alpha\| .
\end{aligned}
$$

Proof. The proof is trivial and can be seen in [13].

The following result describes the local convergence theorem for the iterative method (4)

Theorem 1. Let $F$ be a nonlinear operator satisfying assumptions (5), (6) and (7). Then, the sequence $\left\{x_{k+1}\right\}$ generated by (4) is well defined for $x_{0} \in B\left(\alpha, r_{3}\right)$ and converges to $\alpha$, where, $r_{3}$ is the smallest positive root of $s_{3}$. Also, we obtain the following inequalities for $k \geq 0$ :

$$
\begin{aligned}
\left\|y_{k}-\alpha\right\| & \leq g_{1}\left(\left\|x_{k}-\alpha\right\|\right)\left\|x_{k}-\alpha\right\|<\left\|x_{k}-\alpha\right\|<r_{3}, \\
\left\|z_{k}-\alpha\right\| & \leq g_{2}\left(\left\|x_{k}-\alpha\right\|\right)\left\|x_{k}-\alpha\right\|<\left\|x_{k}-\alpha\right\|<r_{3}, \\
\left\|x_{k+1}-\alpha\right\| & \leq g_{3}\left(\left\|x_{k}-\alpha\right\|\right)\left\|x_{k}-\alpha\right\|<\left\|x_{k}-\alpha\right\|<r_{3},
\end{aligned}
$$

where, $s_{3}, g_{1}, g_{2}$ and $g_{3}$ are auxiliary functions defined in the proof. If there exists a $R \in\left[r_{3}, \frac{2}{L_{0}}\right)$ such that $\bar{B}(\alpha, R) \subseteq D$, then $\alpha$ is the unique solution in $\bar{B}(\alpha, R)$.

Proof. Since $x_{0} \in D$ and using (6), we get

$$
\left\|I-F^{\prime}(\alpha)^{-1} F^{\prime}\left(x_{0}\right)\right\|=\left\|F^{\prime}(\alpha)^{-1}\left(F^{\prime}\left(x_{0}\right)-F^{\prime}(\alpha)\right)\right\| \leq L_{0}\left\|x_{0}-\alpha\right\|<1
$$


for $\left\|x_{0}-\alpha\right\|<\frac{1}{L_{0}}$. Therefore, by Banach Lemma, $F^{\prime}\left(x_{0}\right)^{-1}$ exists and

$$
\left\|F^{\prime}\left(x_{0}\right)^{-1} F^{\prime}(\alpha)\right\| \leq \frac{1}{1-L_{0}\left\|x_{0}-\alpha\right\|} .
$$

Thus, $y_{0}$ is well defined. From the first equation of (4) for $k=0$, we get

$$
\begin{aligned}
y_{0}-\alpha & =x_{0}-\alpha-F^{\prime}\left(x_{0}\right)^{-1} F\left(x_{0}\right) \\
& =-F^{\prime}\left(x_{0}\right)^{-1}\left(F\left(x_{0}\right)-F^{\prime}\left(x_{0}\right)\left(x_{0}-\alpha\right)\right) \\
& =-F^{\prime}\left(x_{0}\right)^{-1} F^{\prime}(\alpha) \int_{0}^{1} F^{\prime}(\alpha)^{-1}\left[F^{\prime}\left(\alpha+t\left(x_{0}-\alpha\right)\right)-F^{\prime}\left(x_{0}\right)\right]\left(x_{0}-\alpha\right) d t
\end{aligned}
$$

By using adequately Banach Lemma, the assumptions and denoting $e_{0}=\left\|x_{0}-\alpha\right\|$, we have

$$
\left\|y_{0}-\alpha\right\| \leq \frac{L\left\|x_{0}-\alpha\right\|}{2\left(1-L_{0}\left\|x_{0}-\alpha\right\|\right)}\left\|x_{0}-\alpha\right\| \leq g_{1}\left(e_{0}\right) e_{0}
$$

where,

$$
g_{1}(t)=\frac{L t}{2\left(1-L_{0} t\right)} .
$$

Obviously $g_{1}$ is an increasing function, and by taking $r_{1}=\frac{2}{L+2 L_{0}}$ it follows:

$$
0 \leq g_{1}(t)<1, \forall t \in\left[0, r_{1}\right) \text {. }
$$

Using (12) and (13), we get

$$
\left\|y_{0}-\alpha\right\| \leq g_{1}\left(\left\|x_{0}-\alpha\right\|\right)\left\|x_{0}-\alpha\right\|<\left\|x_{0}-\alpha\right\| .
$$

Again using (4) for $k=0$, we get

$$
\begin{aligned}
z_{0}-\alpha & =x_{0}-\alpha-2\left(F^{\prime}\left(y_{0}\right)+F^{\prime}\left(x_{0}\right)\right)^{-1} F\left(x_{0}\right) \\
& =\left(F^{\prime}\left(y_{0}\right)+F^{\prime}\left(x_{0}\right)\right)^{-1}\left[\left(F^{\prime}\left(y_{0}\right)+F^{\prime}\left(x_{0}\right)\right)\left(x_{0}-\alpha\right)-2 F\left(x_{0}\right)\right] \\
& =-\left(F^{\prime}\left(y_{0}\right)+F^{\prime}\left(x_{0}\right)\right)^{-1}\left[F\left(x_{0}\right)-F^{\prime}\left(x_{0}\right)\left(x_{0}-\alpha\right)+F\left(x_{0}\right)-F^{\prime}\left(y_{0}\right)\left(x_{0}-\alpha\right)\right] \\
& =-\left(F^{\prime}\left(y_{0}\right)+F^{\prime}\left(x_{0}\right)\right)^{-1} F^{\prime}(\alpha)\left[2 F^{\prime}(\alpha)^{-1} \int_{0}^{1}\left[F^{\prime}\left(\alpha+t\left(x_{0}-\alpha\right)\right)-F^{\prime}\left(x_{0}\right)\right]\left(x_{0}-\alpha\right) d t\right. \\
& \left.+F^{\prime}(\alpha)^{-1}\left(F^{\prime}\left(x_{0}\right)-F^{\prime}\left(y_{0}\right)\right)\left(x_{0}-\alpha\right)\right] .
\end{aligned}
$$

To follow with the study of existence and bound for the product $\left(F^{\prime}\left(y_{0}\right)+F^{\prime}\left(x_{0}\right)\right)^{-1} F^{\prime}(\alpha)$ we observe that

$$
-\frac{1}{2} F^{\prime}(\alpha)^{-1}\left[F^{\prime}\left(x_{0}\right)+F^{\prime}\left(y_{0}\right)-2 F^{\prime}(\alpha)\right]=I-\underbrace{\frac{1}{2} F^{\prime}(\alpha)^{-1}\left[F^{\prime}\left(x_{0}\right)+F^{\prime}\left(y_{0}\right)\right]}_{A}=I-A,
$$


so we try to apply Banach Lemma

$$
\begin{aligned}
\|I-A\| & =\left\|\frac{1}{2} F^{\prime}(\alpha)^{-1}\left[F^{\prime}\left(x_{0}\right)+F^{\prime}\left(y_{0}\right)-2 F^{\prime}(\alpha)\right]\right\| \\
& =\left\|\frac{1}{2}\left[F^{\prime}(\alpha)^{-1}\left(F^{\prime}\left(x_{0}\right)-F^{\prime}(\alpha)\right)+F^{\prime}(\alpha)^{-1}\left(F^{\prime}\left(y_{0}\right)-F^{\prime}(\alpha)\right)\right]\right\| \\
& \leq \frac{1}{2}\left(L_{0}\left\|x_{0}-\alpha\right\|+L_{0}\left\|y_{0}-\alpha\right\|\right) \\
& \leq \frac{1}{2}\left(L_{0} e_{0}+L_{0} g_{1}\left(e_{0}\right) e_{0}\right)=p_{1}\left(e_{0}\right)
\end{aligned}
$$

where $p_{1}(t)=\frac{1}{2} L_{0}\left(1+g_{1}(t)\right) t$ is an increasing function such that $p_{1}(0)=0$ and $p_{1}\left(r_{1}\right)=\frac{1}{2} L_{0} r_{1}\left(1+g_{1}\left(r_{1}\right)\right)=$ $L_{0} r_{1}<1$ and so one has:

$$
\left\|2\left(F^{\prime}\left(x_{0}\right)+F^{\prime}\left(y_{0}\right)\right)^{-1} F^{\prime}(\alpha)^{-1}\right\| \leq \frac{1}{1-p_{1}\left(e_{0}\right)} .
$$

Then, turning to the expression of $z_{0}-\alpha$ we have

$$
\begin{aligned}
\left\|z_{0}-\alpha\right\| & \leq \frac{1}{2\left(1-p\left(\left\|x_{0}-\alpha\right\|\right)\right)}\left[2 L \int_{0}^{1}\left\|\alpha+t\left(x_{0}-\alpha\right)-x_{0}\right\|\left\|x_{0}-\alpha\right\| d t+L\left\|x_{0}-y_{0}\right\|\left\|x_{0}-\alpha\right\|\right] \\
& \leq \frac{1}{2\left(1-p\left(\left\|x_{0}-\alpha\right\|\right)\right)}\left[2 L \frac{\left\|x_{0}-\alpha\right\|^{2}}{2}+L\left(\left\|x_{0}-\alpha\right\|+\left\|y_{0}-\alpha\right\|\right)\left\|x_{0}-\alpha\right\|\right] \\
& \leq \frac{1}{2\left(1-p\left(e_{0}\right)\right)}\left[L e_{0}+L\left(e_{0}+g_{1}\left(e_{0}\right) e_{0}\right)\right] e_{0} \\
& \leq \frac{L e_{0}\left(2+g_{1}\left(e_{0}\right)\right)}{2\left(1-p\left(e_{0}\right)\right)} e_{0}=g_{2}\left(e_{0}\right) e_{0}
\end{aligned}
$$

where

$$
g_{2}(t)=\frac{L t\left(2+g_{1}(t)\right)}{2\left(1-p_{1}(t)\right)} .
$$

So, we consider $s_{2}(t)=g_{2}(t)-1$ having that $s_{2}(0)=-1$ and $s_{2}\left(r_{1}\right)>0$. Therefore, $s_{2}(t)$ has at least one root in $\left(0, r_{1}\right)$ and let $r_{2}$ be the smallest one. Therefore, $0<r_{2}<r_{1}$ and

$$
0 \leq g_{2}(t) \leq 1 \forall t \in\left[0, r_{2}\right),
$$

then by using (14) and (15), we get

$$
\left\|z_{0}-\alpha\right\| \leq g_{2}\left(\left\|x_{0}-\alpha\right\|\right)\left\|x_{0}-\alpha\right\|<\left\|x_{0}-\alpha\right\| .
$$

Again using (4) for $k=0$, we get

$$
x_{1}-\alpha=z_{0}-\alpha-F^{\prime}\left(y_{0}\right)^{-1} F^{\prime}(\alpha) F^{\prime}(\alpha)^{-1} F\left(z_{0}\right)
$$

Since $y_{0} \in D$ and using (6), we get

$$
\begin{aligned}
\left\|I-F^{\prime}(\alpha)^{-1} F^{\prime}\left(y_{0}\right)\right\| & \leq\left\|F^{\prime}(\alpha)^{-1}\left(F^{\prime}(\alpha)-F^{\prime}\left(y_{0}\right)\right)\right\| \\
& \leq L_{0}\left\|y_{0}-\alpha\right\| \leq L_{0} g_{1}\left(e_{0}\right) e_{0}=p_{2}\left(e_{0}\right)<1
\end{aligned}
$$


where

$$
p_{2}(t)=L_{0} g_{1}(t) t
$$

Then $\exists\left(F^{\prime}(\alpha)^{-1} F^{\prime}\left(y_{0}\right)\right)^{-1}$ and

$$
\left\|F^{\prime}\left(y_{0}\right)^{-1} F^{\prime}(\alpha)\right\| \leq \frac{1}{1-p_{2}\left(e_{0}\right)} .
$$

Therefore, by using Lemma 1, we get

$$
\begin{aligned}
\left\|x_{1}-\alpha\right\| & \leq\left\|z_{0}-\alpha\right\|+\frac{1}{1-p_{2}\left(e_{0}\right)}\left(1+L_{0}\left\|z_{0}-\alpha\right\|\right)\left\|z_{0}-\alpha\right\| \\
& =\left(1+\frac{1+L_{0} g_{2}\left(e_{0}\right) e_{0}}{1-p_{2}\left(e_{0}\right)}\right) g_{2}\left(e_{0}\right) e_{0}=g_{3}\left(e_{0}\right) e_{0},
\end{aligned}
$$

where

$$
g_{3}(t)=\left(1+\frac{1+L_{0} g_{2}(t) t}{1-p_{2}(t)}\right) g_{2}(t) .
$$

Consider $s_{3}(t)=g_{3}(t)-1$. Then, $s_{3}(0)=-1$ and $s_{3}\left(r_{2}\right)>0$. Therefore, $s_{3}(t)$ has at least one root in $\left(0, r_{2}\right)$ and let $r_{3}$ the smallest one. Therefore, $0<r_{3}<r_{2}$

$$
0 \leq g_{3}(t) \leq 1 \forall t \in\left[0, r_{3}\right)
$$

then by using (16) and (17), we get

$$
\left\|x_{1}-\alpha\right\| \leq g_{3}\left(\left\|x_{0}-\alpha\right\|\right)\left\|x_{0}-\alpha\right\|<\left\|x_{0}-\alpha\right\|<\eta .
$$

Thus, Theorem 1 holds for $k=0$. Changing $x_{0}, y_{0}, z_{0}$ and $x_{1}$ by $x_{k}, y_{k}, z_{k}, x_{k+1}$, we get the inequalities (8)-(10) for all $k \geq 0$. Since, $\left\|x_{k+1}-\alpha\right\| \leq\left\|x_{k}-\alpha\right\|<r_{3}$, this gives $x_{k+1} \in B\left(\alpha, r_{3}\right)$. Also $g_{3}(t)$ is an increasing function in $\left[0, r_{3}\right)$, since $g_{3}^{\prime}(t)>0$ for all $t \in\left[0, r_{3}\right)$. Thus, we get

$$
\begin{aligned}
\left\|x_{k+1}-\alpha\right\| & \leq g_{3}\left(e_{0}\right)\left\|x_{k}-\alpha\right\| \leq g_{3}\left(e_{0}\right) g_{3}\left(e_{0}\right)\left\|x_{k-1}-\alpha\right\| \\
& \leq g_{3}\left(e_{0}\right)^{2} g_{3}\left(e_{0}\right)\left\|x_{k-2}-\alpha\right\| \leq \ldots \leq g_{3}\left(e_{0}\right)^{k+1}\left\|x_{0}-\alpha\right\| .
\end{aligned}
$$

Therefore, $\lim _{k \rightarrow \infty} x_{k}=\alpha$ as $g_{3}(t)<1$.

For getting the uniqueness ball for root $\alpha$, let $\beta \in B(\alpha, R)$ be such that $F(\beta)=0$ and $\beta \neq \alpha$. Consider $P=\int_{0}^{1} F^{\prime}(\beta+t(\alpha-\beta)) d t$. Using (6), we get

$$
\left\|F^{\prime}(\alpha)^{-1}\left(P-F^{\prime}(\alpha)\right)\right\| \leq \int_{0}^{1} L_{0}\|\beta+t(\alpha-\beta)-\alpha\| d t \leq \frac{L_{0}}{2}\|\alpha-\beta\|=\frac{L_{0}}{2} R<1,
$$

therefore, by Banach Lemma, $P^{-1}$ exists. Then,

$$
0=F(\alpha)-F(\beta)=P(\alpha-\beta),
$$

we obtain $\alpha=\beta$. 


\subsection{Numerical examples}

In this subsection, we consider numerical examples to demonstrate the applicability of our work. Moreover, we compare our results with the local convergence of a modified Halley-Like method (2) and Chebyshev-Halley-type methods (3) respectively.

Example 1. Let $X=Y=\mathbb{R}^{3}, D=\bar{U}(0,1)$. Define $F$ on $D$ for $v=(x, y, z)$ by

$$
F(v)=\left(e^{x}-1, \frac{e-1}{2} y^{2}+y, z\right) .
$$

Clearly, $\alpha=(0,0,0), F^{\prime}(\alpha)=F^{\prime}(\alpha)^{-1}=\operatorname{diag}\{1,1,1\}, L_{0}=e-1$, and $L=e$. Then, we have

$$
r_{3}=0.13125<r_{2}=0.21657<r_{1}=0.32495 \text {. }
$$

Example 2. Consider the system of nonlinear equations

$$
\begin{aligned}
& 2 x_{1}-\frac{1}{9} x_{1}^{2}-x_{2}=0 \\
& -x_{1}+2 x_{2}-\frac{1}{9} x_{2}^{2}=0
\end{aligned}
$$

The associated nonlinear operator $F: R^{2} \rightarrow R^{2}$ is given by

$$
F\left(x_{1}, x_{2}\right)=\left(\begin{array}{l}
F_{1}\left(x_{1}, x_{2}\right) \\
F_{2}\left(x_{1}, x_{2}\right)
\end{array}\right)
$$

where $F_{1}\left(x_{1}, x_{2}\right)=2 x_{1}-\frac{1}{9} x_{1}^{2}-x_{2}$ and $F_{2}\left(x_{1}, x_{2}\right)=-x_{1}+2 x_{2}-\frac{1}{9} x_{2}^{2}$.

Clearly $\alpha=(9,9)^{T}$ is a solution of above nonlinear system and for all $(x, y) \in R^{2}$ we have:

$$
\begin{aligned}
\left\|F^{\prime}(\alpha)^{-1}\left(F^{\prime}(x)-F^{\prime}(y)\right)\right\| & =\frac{2}{9}\|x-y\| \\
\left\|F^{\prime}(\alpha)^{-1}\left(F^{\prime}(x)-F^{\prime}(\alpha)\right)\right\| & =\frac{2}{9}\|x-\alpha\| .
\end{aligned}
$$

Taking $L_{0}=\frac{2}{9}$ and $L=\frac{2}{9}$, we get

$$
r_{3}=1.44284<r_{2}=2.25000<r_{1}=3.00000 .
$$

Example 3. Consider the nonlinear Hammerstein type integral equation given by

$$
F(x(s))=x(s)-5 \int_{0}^{1} s t x(t)^{3} d t
$$

with $x(s)$ in $\mathcal{C}[0,1]$.

Clearly $\alpha=0$. Taking $L_{0}=7.5$ and $L=15$, we get

$$
r_{3}=0.02481<r_{2}=0.04185<r_{1}=0.06667 \text {. }
$$

Now, we compare our results with the local convergence of a modified Halley-Like method (2) and Chebyshev-Halley-type methods (3) respectively. The value of parameters used by these methods are listed in Table 1. The radius of a convergence ball of a fifth order method (4) is compared with method (2) and method (3) in Table 2. We can observe that the larger radius of convergence ball is obtained by our approach. 
Table 1: Values of parameters

\begin{tabular}{llll}
\hline Examples & $a$ & $\gamma$ & $\eta$ \\
\hline 1 & 1.0125 & 0.3 & 0.03 \\
2 & 1 & $1 / 9$ & $2 / 9$ \\
3 & 1 & 0.575 & 0.003 \\
\hline
\end{tabular}

Table 2: Comparison of radius of a ball

\begin{tabular}{llll}
\hline Examples & Method (4) & Method (2) & Method (3) \\
\hline 1 & 0.13125 & 0.02726 & 0.00892 \\
2 & 1.44284 & 0.55264 & 0.06989 \\
3 & 0.02481 & 0.00709 & 0.00755 \\
\hline
\end{tabular}

\section{The derivative free method and its local convergence analysis}

In this section our purpose is to complete the study of iterative method (4), when we use adequate approximation of the derivatives by divided differences. So, now the aim is to obtain the local convergence study in this case.

In order to obtain derivative free iterative methods we approximate derivatives by divided differences. That is an operator $[x, y ; F]$ verifying

$$
[x, y ; F](x-y)=F(x)-F(y), \text { forall } x, y \in D
$$

and if $F$ is Fréchet differentiable at $a \in D$ then $[\alpha, \alpha ; F]=F^{\prime}(\alpha)$. One can see different approximations of divided differences in $[18,19]$.

We consider the derivative free iterative method given for $k=0,1,2, \ldots$ by

$$
\begin{aligned}
y_{k} & =x_{k}-\left[x_{k}, x_{k}+F\left(x_{k}\right) ; F\right]^{-1} F\left(x_{k}\right) \\
z_{k} & =x_{k}-2\left(\left[x_{k}, x_{k}+F\left(x_{k}\right) ; F\right]+\left[y_{k}, y_{k}+F\left(y_{k}\right) ; F\right]\right)^{-1} F\left(x_{k}\right) \\
x_{k+1} & =z_{k}-\left[y_{k}, y_{k}+F\left(y_{k}\right) ; F\right]^{-1} F\left(z_{k}\right),
\end{aligned}
$$

where $x_{0}$ is the starting point.

We use the following assumptions for setting the local convergence study in this case. Let $K_{0}>0$, $K>0$ and for all $x, y, u, v \in D$, we have $F(\alpha)=0, F^{\prime}(\alpha)^{-1} \neq 0$, in $D$, moreover

$$
\begin{aligned}
\left\|F^{\prime}(\alpha)^{-1}([x, y ; F]-[u, v ; F])\right\| & \leq K(\|x-u\|+\|y-v\|), \\
\left\|F^{\prime}(\alpha)^{-1}([x, y ; F]-[\alpha, \alpha ; F])\right\| & \leq K_{0}(\|x-\alpha\|+\|y-\alpha\|), \\
\|F(x)-F(\alpha)\| & \leq L\|x-\alpha\| .
\end{aligned}
$$

The next result describes the local convergence theorem for the derivative free iterative method (19)

Theorem 2. Let $F$ the nonlinear operator satisfying assumptions (20), (21) and (22). Then, the sequence $\left\{x_{k+1}\right\}$ generated by (19) is well defined for any starting point $x_{0} \in B\left(\alpha, \rho_{3}\right)$ and converges to $\alpha$, where $\rho_{3}$ is the smallest positive root of function $q_{3}$. Also, we obtain the following inequalities for $k \geq 0$ : 


$$
\begin{aligned}
\left\|y_{k}-\alpha\right\| & \leq h_{1}\left(\left\|x_{k}-\alpha\right\|\right)\left\|x_{k}-\alpha\right\|<\left\|x_{k}-\alpha\right\|<\rho_{3}, \\
\left\|z_{k}-\alpha\right\| & \leq h_{2}\left(\left\|x_{k}-\alpha\right\|\right)\left\|x_{k}-\alpha\right\|<\left\|x_{k}-\alpha\right\|<\rho_{3}, \\
\left\|x_{k+1}-\alpha\right\| & \leq h_{3}\left(\left\|x_{k}-\alpha\right\|\right)\left\|x_{k}-\alpha\right\|<\left\|x_{k}-\alpha\right\|<\rho_{3},
\end{aligned}
$$

where, $h_{1}, h_{2}, h_{3}, q_{3}$ are auxiliary functions defined in the proof and $\rho_{3}$ is the smallest root of $q_{3}(t)$. Moreover, if there exists a $R_{1} \in\left[\rho_{3}, \frac{1}{K_{0}}\right)$ such that $\bar{B}\left(\alpha, R_{1}\right) \subseteq D$, then $\alpha$ is the unique solution in $\bar{B}\left(\alpha, R_{1}\right)$.

Proof. Since $x_{0} \in D$ and using (21), we get

$$
\begin{aligned}
\left\|F^{\prime}(\alpha)^{-1}\left(\left[x_{0}, x_{0}+F\left(x_{0}\right) ; F\right]-[\alpha, \alpha ; F]\right)\right\| & \leq K_{0}\left(\left\|x_{0}-\alpha\right\|+\left\|x_{0}+F\left(x_{0}\right)-\alpha\right\|\right. \\
& \leq K_{0}\left(\left\|x_{0}-\alpha\right\|+(1+L)\left\|x_{0}-\alpha\right\|\right) \\
& =K_{0}(2+L)\left\|x_{0}-\alpha\right\|<1
\end{aligned}
$$

for $\left\|x_{0}-\alpha\right\|<\frac{1}{K_{0}(2+L)}$. Therefore, by Banach Lemma, $\left[x_{0}, x_{0}+F\left(x_{0}\right) ; F\right]^{-1}$ exists and

$$
\left\|\left[x_{0}, x_{0}+F\left(x_{0}\right) ; F\right]^{-1} F^{\prime}(\alpha)\right\| \leq \frac{1}{1-K_{0}(2+L)\left\|x_{0}-\alpha\right\|} .
$$

Thus, $y_{0}$ is well defined. Using (19) for $k=0$, we get

$$
\begin{aligned}
y_{0}-\alpha & =x_{0}-\alpha-\left[x_{0}, x_{0}+F\left(x_{0}\right) ; F\right]^{-1} F\left(x_{0}\right) \\
& =\left[x_{0}, x_{0}+F\left(x_{0}\right) ; F\right]^{-1} F^{\prime}(\alpha) F^{\prime}(\alpha)^{-1}\left(\left[x_{0}, x_{0}+F\left(x_{0}\right) ; F\right]-\left[x_{0}, \alpha ; F\right]\right)\left(x_{0}-\alpha\right) .
\end{aligned}
$$

Using (20), we have

$$
\left\|F^{\prime}(\alpha)^{-1}\left(\left[x_{0}, x_{0}+F\left(x_{0}\right) ; F\right]-\left[x_{0}, \alpha ; F\right]\right)\right\| \leq K\left(\left\|x_{0}+F\left(x_{0}-\alpha \|\right) \leq K(1+L)\right\| x_{0}-\alpha \| .\right.
$$

Therefore,

$$
\begin{aligned}
\left\|y_{0}-\alpha\right\| & \leq\left(\frac{K(1+L)\left\|x_{0}-\alpha\right\|}{1-K_{0}(2+L)\left\|x_{0}-\alpha\right\|}\right)\left\|x_{0}-\alpha\right\| \\
& =h_{1}\left(e_{0}\right) e_{0},
\end{aligned}
$$

where, $h_{1}(t)=\frac{K(1+L) t}{1-K_{0}(2+L) t}$ and $e_{0}=\left\|x_{0}-\alpha\right\|$.

Consider the function $q_{1}(t)=h_{1}(t)-1$. Then, $q_{1}(0)=-1$ and $q_{1}\left(\frac{1}{K_{0}(2+L)}\right) \rightarrow+\infty$. Therefore, $q_{1}(t)$ has atleast one root in $\left(0, \frac{1}{K_{0}(2+L)}\right)$ and let $\rho_{1}$ be such a smallest root. Therefore, $0<\rho_{1}<\frac{1}{K_{0}(2+L)}$, and

$$
0 \leq h_{1}(t)<1 \forall t \in\left[0, \rho_{1}\right) .
$$

Using (27) and (28), we get

$$
\left\|y_{0}-\alpha\right\| \leq h_{1}\left(\left\|x_{0}-\alpha\right\|\right)\left\|x_{0}-\alpha\right\|<\left\|x_{0}-\alpha\right\| .
$$


Again using (19) for $k=0$, we get

$$
\begin{aligned}
z_{0}-\alpha & =x_{0}-\alpha-2\left(\left[x_{0}, x_{0}+F\left(x_{0}\right) ; F\right]+\left[y_{0}, y_{0}+F\left(y_{0}\right) ; F\right]\right)^{-1} F\left(x_{0}\right) \\
& =\left(\left[x_{0}, x_{0}+F\left(x_{0}\right) ; F\right]+\left[y_{0}, y_{0}+F\left(y_{0}\right) ; F\right]\right)^{-1} F^{\prime}(\alpha) F^{\prime}(\alpha)^{-1}\left(\left[x_{0}, x_{0}+F\left(x_{0}\right) ; F\right]\right. \\
& \left.+\left[y_{0}, y_{0}+F\left(y_{0}\right) ; F\right]-2\left[x_{0}, \alpha ; F\right]\right)\left(x_{0}-\alpha\right) .
\end{aligned}
$$

Now we study the existence and bound for the product $\left(\left[x_{0}, x_{0}+F\left(x_{0}\right) ; F\right]+\left[y_{0}, y_{0}+F\left(y_{0}\right) ; F\right]\right)^{-1} F^{\prime}(\alpha)$. Then we observe that

$$
\begin{aligned}
& \frac{1}{2} F^{\prime}(\alpha)^{-1}\left(\left(\left[x_{0}, x_{0}+F\left(x_{0}\right) ; F\right]+\left[y_{0}, y_{0}+F\left(y_{0}\right) ; F\right]\right)-2 F^{\prime}(\alpha)\right) \\
= & I-\underbrace{\frac{1}{2} F^{\prime}(\alpha)^{-1}\left[\left(\left[x_{0}, x_{0}+F\left(x_{0}\right) ; F\right]+\left[y_{0}, y_{0}+F\left(y_{0}\right) ; F\right]\right)\right]}_{C}=I-C .
\end{aligned}
$$

So we try to apply Banach Lemma

$$
\begin{aligned}
\|I-C\| & \leq \frac{1}{2}\left(\left\|F^{\prime}(\alpha)^{-1}\left(\left[x_{0}, x_{0}+F\left(x_{0}\right) ; F\right]-F^{\prime}(\alpha)\right)\right\|+\left\|F^{\prime}(\alpha)^{-1}\left(\left[y_{0}, y_{0}+F\left(y_{0}\right) ; F\right]-F^{\prime}(\alpha)\right)\right\|\right) \\
& \leq \frac{K_{0}}{2}\left(\left(\left\|x_{0}-\alpha\right\|+\left\|x_{0}+F\left(x_{0}\right)-\alpha\right\|\right)+\left(\left\|y_{0}-\alpha\right\|+\left\|y_{0}+F\left(y_{0}\right)-\alpha\right\|\right)\right) \\
& \leq \frac{K_{0}}{2}\left((2+L)\left\|x_{0}-\alpha\right\|+(2+L)\left\|y_{0}-\alpha\right\|\right) \\
& \leq \frac{K_{0}}{2}\left((2+L)\left\|x_{0}-\alpha\right\|+(2+L) h_{1}\left(\left\|x_{0}-\alpha\right\|\right)\left\|x_{0}-\alpha\right\|\right) \\
& =\phi_{1}\left(e_{0}\right),
\end{aligned}
$$

where $\phi_{1}(t)=\frac{K_{0}}{2}(2+L)\left(1+h_{1}(t)\right) t$ is an increasing function such that $\phi_{1}(0)=0$ and $\phi_{1}\left(\rho_{1}\right)=$ $\frac{K_{0}}{2}(2+L) \rho_{1}\left(1+h_{1}\left(\rho_{1}\right)\right)=\frac{K_{0}}{2}(2+L) \rho_{1}<1$ and so we have

$$
\left\|C^{-1}\right\|=\left\|2\left(\left[x_{0}, x_{0}+F\left(x_{0}\right) ; F\right]+\left[y_{0}, y_{0}+F\left(y_{0}\right) ; F\right]\right)^{-1} F^{\prime}(\alpha)\right\| \leq \frac{1}{1-\phi_{1}\left(e_{0}\right)}
$$

Therefore,

$$
\begin{gathered}
\left\|z_{0}-\alpha\right\| \leq \frac{K}{2\left(1-\phi_{1}\left(\left\|x_{0}-\alpha\right\|\right)\right)}\left(\left\|x_{0}+F\left(x_{0}\right)-\alpha\right\|+\left\|y_{0}-x_{0}\right\|+\left\|y_{0}+F\left(y_{0}\right)-\alpha\right\|\right)\left\|x_{0}-\alpha\right\| \\
\leq \frac{K}{2\left(1-\phi_{1}\left(e_{0}\right)\right)}\left((1+L) e_{0}+\left(1+h_{1}\left(e_{0}\right)\right) e_{0}+(1+L) h_{1}\left(e_{0}\right) e_{0}\right) e_{0} \\
\leq \frac{K}{2\left(1-\phi_{1}\left(e_{0}\right)\right)}( \\
=h_{2}\left(e_{0}\right) e_{0},
\end{gathered}
$$

where

$$
h_{2}(t)=\frac{K}{2\left(1-\phi_{1}(t)\right)}\left((2+L)\left(1+h_{1}(t)\right) t\right) .
$$

Consider $q_{2}(t)=h_{2}(t)-1$. Then, $q_{2}(0)=-1$ and $q_{2}\left(\rho_{1}\right)=\frac{K \rho_{1}(2+L)}{1-K_{0}(2+L) \rho_{1}}>0$. Therefore, $q_{2}(t)$ has atleast one root in $\left(0, \rho_{1}\right)$ and let $\rho_{2}$ be such a smallest root. Therefore, $0<\rho_{2}<\rho_{1}$ and

$$
0 \leq h_{2}(t) \leq 1 \forall t \in\left[0, \rho_{2}\right)
$$


Using (29) and (30), we get

$$
\left\|z_{0}-\alpha\right\| \leq h_{2}\left(\left\|x_{0}-\alpha\right\|\right)\left\|x_{0}-\alpha\right\|<\left\|x_{0}-\alpha\right\| .
$$

Taking $k=0$ in (19), we get

$$
x_{1}-\alpha=z_{0}-\alpha-\left[y_{0}, y_{0}+F\left(y_{0}\right) ; F\right]^{-1} F^{\prime}(\alpha) F^{\prime}(\alpha)^{-1} F\left(z_{0}\right) .
$$

Since $y_{0} \in D$, we have

$$
\begin{aligned}
\left\|F^{\prime}(\alpha)^{-1}\left(\left[y_{0}, y_{0}+F\left(y_{0}\right) ; F\right]-[\alpha, \alpha ; F]\right)\right\| & \leq K_{0}\left(\left\|y_{0}-\alpha\right\|+\left\|y_{0}+F\left(y_{0}\right)-\alpha\right\|\right. \\
& \leq K_{0}\left(\left\|y_{0}-\alpha\right\|+(1+L)\left\|y_{0}-\alpha\right\|\right) \\
& \leq K_{0}(2+L) h_{1}\left(e_{0}\right) e_{0}=\phi_{2}\left(e_{0}\right)<1
\end{aligned}
$$

where $\phi_{2}(t)=K_{0}(2+L) h_{1}(t) t$. Thus, by Banach Lemma, we have

$$
\left\|\left[y_{0}, y_{0}+F\left(y_{0}\right) ; F\right]^{-1} F^{\prime}(\alpha)\right\| \leq \frac{1}{1-\phi_{2}\left(e_{0}\right)} .
$$

Therefore,

$$
\begin{aligned}
\left\|x_{1}-\alpha\right\| & \leq\left\|\left[y_{0}, y_{0}+F\left(y_{0}\right) ; F\right]^{-1} F^{\prime}(\alpha)\right\|\left\|F^{\prime}(\alpha)^{-1}\left(\left[y_{0}, y_{0}+F\left(y_{0}\right) ; F\right]-\left[z_{0}, \alpha ; F\right]\right)\right\|\left\|z_{0}-\alpha\right\| \\
& \leq \frac{K\left(\left\|y_{0}-z_{0}\right\|+\left\|y_{0}+F\left(y_{0}\right)-\alpha\right\|\right)}{1-\phi_{2}\left(e_{0}\right)}\left\|z_{0}-\alpha\right\| .
\end{aligned}
$$

As

$$
\begin{gathered}
\left\|y_{0}-z_{0}\right\| \leq\left\|y_{0}-\alpha\right\|+\left\|z_{0}-\alpha\right\| \leq\left(h_{1}(t)+h_{2}(t)\right)\left\|x_{0}-\alpha\right\| . \\
\left\|x_{1}-\alpha\right\|=\frac{K\left(\left(h_{1}\left(e_{0}\right)+h_{2}\left(e_{0}\right)\right) e_{0}+(1+L) h_{1}\left(e_{0}\right) e_{0}\right)}{1-\phi_{2}\left(e_{0}\right)} h_{2}\left(e_{0}\right)\left\|x_{0}-\alpha\right\| \\
=h_{3}\left(e_{0}\right) e_{0} .
\end{gathered}
$$

where

$$
h_{3}(t)=\left(\frac{K\left((2+L) h_{1}(t) t+h_{2}(t) t\right)}{1-\phi_{2}(t)}\right) h_{2}(t) .
$$

Consider $q_{3}(t)=h_{3}(t)-1$. Then, $q_{3}(0)=-1$ and $q_{3}\left(\rho_{2}\right)>0$. Therefore, $q_{3}(t)$ has at least one root in $\left(0, \rho_{2}\right)$ and let $\rho_{3}$ be such a smallest root. Therefore, $0<\rho_{3}<\rho_{2}$, and

$$
0 \leq h_{3}(t) \leq 1 \forall t \in\left[0, \rho_{3}\right)
$$

Using (31) and (32), we get

$$
\left\|x_{1}-\alpha\right\| \leq h_{3}\left(\left\|x_{0}-\alpha\right\|\right)\left\|x_{0}-\alpha\right\|<\left\|x_{0}-\alpha\right\|
$$

Thus, theorem holds for $k=0$. Changing $x_{0}, y_{0}, z_{0}$ and $x_{1}$ by $x_{k}, y_{k}, z_{k}, x_{k+1}$, we get the inequalities (23)-(25) for all $k \geq 0$. Since, $\left\|x_{k+1}-\alpha\right\| \leq\left\|x_{k}-\alpha\right\|<r_{3}$, this gives $x_{k+1} \in B\left(\alpha, \rho_{3}\right)$. Also $h_{3}(t)$ is an increasing function in $\left[0, \rho_{3}\right)$, since $h_{3}^{\prime}(t)>0$ for all $t \in\left[0, \rho_{3}\right)$. Thus, we get

$$
\begin{aligned}
\left\|x_{k+1}-\alpha\right\| & \leq h_{3}\left(e_{0}\right)\left\|x_{k}-\alpha\right\| \leq h_{3}\left(e_{0}\right) h_{3}\left(e_{0}\right)\left\|x_{k-1}-\alpha\right\| \\
& \leq h_{3}\left(e_{0}\right)^{2} h_{3}\left(e_{0}\right)\left\|x_{k-2}-\alpha\right\| \leq \cdots \leq h_{3}\left(e_{0}\right)^{k+1}\left\|x_{0}-\alpha\right\| .
\end{aligned}
$$


Therefore, $\lim _{k \rightarrow \infty} x_{k}=\alpha$ as $h_{3}(t)<1$.

For uniqueness part, let $P_{1}=[\alpha, \beta ; F]$ where $F(\beta)=0$ and $\beta \in \bar{B}\left(\alpha, R_{1}\right)$. Thus, we have

$$
\left\|F^{\prime}(\alpha)^{-1}\left(P_{1}-F^{\prime}(\alpha)\right)\right\| \leq K_{0}(\|\alpha-\alpha\|+\|\beta-\alpha\|) \leq K_{0} R_{1}<1,
$$

therefore, by Banach Lemma, $P_{1}^{-1}$ exists. Then,

$$
0=F(\alpha)-F(\beta)=P_{1}(\alpha-\beta)
$$

we obtain $\alpha=\beta$.

\subsection{Numerical examples}

In this subsection, we consider numerical examples to demonstrate the applicability of our work. Moreover, we compare our results with the local convergence of a modified Halley-Like method (2) and Chebyshev-Halley-type methods (3) respectively.

Example 4. Let $X=Y=\mathbb{R}, D=(-1,1)$. Define $F$ on $D$ by

$$
F(x)=e^{x}-1 .
$$

Clearly, $\alpha=0, F^{\prime}(\alpha)=F^{\prime}(\alpha)^{-1}=1, K_{0}=\frac{e-1}{2}$, and $L=e$. Then, we have

$$
r_{3}=0.100343<r_{2}=0.101834<r_{1}=0.109801 \text {. }
$$

Example 5. Let $X=Y=\mathbb{R}, D=(-1,1)$. Define $F$ on $D$ by

$$
F(x)=x^{2}-1 .
$$

Clearly, $\alpha=1, K_{0}=K=\frac{1}{2}$, and $L=2$. Then, we have

$$
r_{3}=0.265055<r_{2}=0.267949<r_{1}=0.285714 \text {. }
$$

Example 6. Consider the nonlinear Hammerstein type integral equation given by

$$
F(x(s))=x(s)-5 \int_{0}^{1} s t x(t)^{3} d t
$$

with $x(s)$ in $\mathcal{C}[0,1]$.

Clearly $\alpha=0$. Taking $K_{0}=3.75, K=7.5$ and $L=8.5$ we get

$$
r_{3}=0.008636<r_{2}=0.008708<r_{1}=0.009039 \text {. }
$$

The value of parameters are listed in Table 1. The radius of a convergence ball of a derivative free fifth order method (19) is compared with the existing methods and showed in Table 3. We can observe that except in example 5, all other examples larger radius of convergence ball is obtained by our approach. In example 5, we observe that the larger radius of convergence is obtained as compared to the Method (3). 
Table 3: Comparison of radius of a ball

\begin{tabular}{llll}
\hline Examples & Method (19) & Method (2) & Method (3) \\
\hline 4 & 0.100343 & 0.02726 & 0.00892 \\
5 & 0.265055 & 0.55264 & 0.06989 \\
6 & 0.008636 & 0.00709 & 0.00755 \\
\hline
\end{tabular}

\section{Conclusions}

In this paper, we discussed the local convergence of two fifth order iterative methods for solving nonlinear equations in Banach spaces, including the corresponding study when we consider the derivative free method obtained by approximating the derivatives by divided difference, getting a complete analysis of this iterative method. This analysis established under the assumption that the first order Fréchet derivative satisfies the Lipschitz continuity condition for first case and similar condition for the derivative free method involving only the derivative at the exact solution. Finally, for each method, some numerical examples worked out and computed the radii of convergence. Also, we have compared these results with existing methods and observed that our results are more efficient.

\section{References}

[1] A. Constantinides, N. Mostoufi, Numerical Methods for Chemical Engineers with MATLAB Applications, Prentice Hall PTR, New Jersey, (1999).

[2] J.M. Douglas, Process Dynamics and Control, Prentice Hall, Englewood Cliffs, (1972).

[3] M. Shacham, An improved memory method for the solution of a nonlinear equation, Chem. Eng. Sci., 44 (1989), 1495-1501.

[4] J.M. Ortega, W.C. Rheinboldt, Iterative solution of nonlinear equations in several variables, Academic Press, New-York, (1970).

[5] J.R. Sharma, H. Arora, A novel derivative free algorithm with seventh order convergence for solving systems of nonlinear equations, Numer. Algorithms, 67 (2014), 917-933.

[6] I.K. Argyros, A.A. Magrenan, L. Orcos, Local convergence and a chemical application of derivative free root finding methods with one parameter based on interpolation, J. Math. Chem., 54 (2016), 1404-1416.

[7] E.L. Allgower, K. Georg, Lectures in Applied Mathematics, American Mathematical Society (Providence, RI) 26, 723-762.

[8] A.V. Rangan, D. Cai, L. Tao, Numerical methods for solving moment equations in kinetic theory of neuronal network dynamics, J. Comput. Phys., 221 (2007), 781-798.

[9] A. Nejat, C. Ollivier-Gooch, Effect of discretization order on preconditioning and convergence of a high-order unstructured Newton-GMRES solver for the Euler equations, J. Comput. Phys., 227 (2008), 2366-2386. 
[10] C. Grosan, A. Abraham, A new approach for solving nonlinear equations systems, IEEE Trans. Syst. Man Cybernet Part A: System Humans, 38 (2008), 698-714.

[11] F. Awawdeh, On new iterative method for solving systems of nonlinear equations, Numer. Algorithms, 54 (2010), 395-409.

[12] I.G. Tsoulos, A. Stavrakoudis, On locating all roots of systems of nonlinear equations inside bounded domain using global optimization methods, Nonlinear Anal Real World Appl., 11 (2010), 2465-2471.

[13] E. Martinez, S. Singh, J.L.Hueso, D.K. Gupta, Enlarging the convergence domain in local convergence studies for iterative methods in Banach spaces, Appl. Math. Comput., 281 (2016), 252-265.

[14] S. Singh, D.K. Gupta, E. Martinez, J.L.Hueso, Semi local and local convergence of a fifth order iteration with Fréchet derivative satisfying Hölder condition, Appl. Math. Comput., 276 (2016), 266-277.

[15] I.K. Argyros, S. George, Local convergence of modified Halley-like methods with less computation of inversion, Novi. Sad.J. Math. 45 (2015), 47-58.

[16] A. Cordero, J.L. Hueso, E. Martinez, J.R. Torregrosa, Increasing the convergence order of an iterative method for nonlinear systems, Appl. Math. Lett., 25 (2012), 2369-2374.

[17] I.K. Argyros, A.A. Magreñán, A study on the local convergence and dynamics of ChebyshevHalley-type methods free from second derivative, Numer. Algorithms 71 (2016), 1-23.

[18] M. Grau-Sánchez, Á Grau, M Noguera, Frozen divided difference scheme for solving systems of nonlinear equations, J. Comput. Appl. Math., 235 (2011), 1739-1743.

[19] M. Grau-Sánchez, M. Noguera, S. Amat, On the approximation of derivatives using divided difference operators preserving the local convergence order of iterative methods, J. Comput. Appl. Math., 237 (2013), 363-372. 